\title{
Ultrastructural and biochemical detection of biotin and biotinylated polypeptides in Schistosoma mansoni
}

P.R.P. Santos ${ }^{1}$ and M.E.C. Chaves ${ }^{1,2}$

\author{
'Laboratório de Imunopatologia Keizo Asami (LIKA), \\ Universidade Federal de Pernambuco, 50670-901 Recife, PE, Brasil \\ ${ }^{2}$ Departamento de Bioquímica, Centro de Ciências Biológicas, \\ Universidade Federal de Pernambuco, 50670-420 Recife, PE, Brasil
}

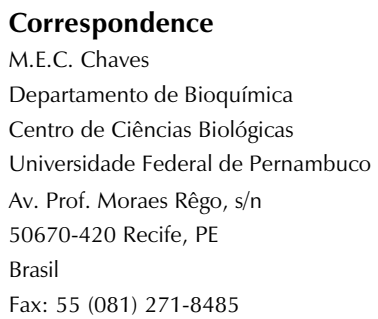

Research supported by the Japan International Cooperation Agency (JICA) and CNPq.

Received April 30, 1996 Accepted May 21, 1997

\begin{abstract}
Biotinylation is proposed for the identification of surface proteins in Schistosoma mansoni using the streptavidin-HRP conjugate for the detection of labeled polypeptides. However, control samples also showed several endogenous biotinylated polypeptides. In an attempt to determine the possibility of nonspecific binding between the streptavidin-HRP conjugate and polypeptides from S. mansoni, the conjugate was blocked with biotinamidecaproate-N-hydroxysuccinimide ester (BcapNHS) before biotin-streptavidin blotting. No bands were detected on the nitrocellulose sheet, demonstrating the specific recognition of biotin by the streptavidin present in the conjugate. Whole cercariae and cercarial bodies and tails showed several endogenous biotinylated polypeptides. The biotin concentration was $13 \mu \mathrm{g} /$ 190,000 cercariae. Adult worms presented less endogenous biotinylated polypeptides than cercariae. These results may be due to changes in the environment from aerobic to anaerobic conditions when cercarial bodies (schistosomula) are transformed into adult worms and a decrease in $\mathrm{CO}_{2}$ production may occur. Cercariae, cercarial bodies and adult male worms were examined by transmission electron microscopy employing an avidin-colloidal gold conjugate for the detection of endogenous biotin. Gold particles were distributed mainly on the muscle fibers, but dispersed granules were observed in the tegument, mitochondria and cytosol. The discovery of endogenous biotin in $S$. mansoni should be investigated in order to clarify the function of this vitamin in the parasite.
\end{abstract}

\author{
Key words \\ - Biotin \\ - Biotinylated polypeptides \\ - Schistosoma mansoni \\ .....................
}

\section{Introduction}

Schistosoma mansoni is one of the most prevalent parasitic helminths that infect humans. Snails of the genus Biomphalaria are the intermediate host of this parasite, releasing cercariae, the infective larvae for humans, into fresh water. During penetration through the skin, the cercariae lose their tails and the glycocalyx, originating the schistosomula, that later will be transformed into the adult worms. At the same time, changes on the surface and ultrastructural and metabolic changes occur to adapt the worms to the new environment.

S. mansoni survival is dependent on the 
degradation of glucose obtained from the endogenous glycogen reserve. Evidence for the presence of gluconeogenesis in S. mansoni was not observed until recently, when the activity of four gluconeogenic enzymes was demonstrated in adult worms of S. mansoni (1). Two of those enzymes were carboxylases which must contain biotin as prosthetic group in their structures. The presence of endogenous biotin has been shown in several prokaryotes (2-4), fungi and yeast $(5,6)$ and in birds and mammals $(7,8)$. However, no reports are available about this vitamin in helminths.

In the present study, we identified endogenous biotinylated polypeptides in cercariae and adult worms of $S$. mansoni using a streptavidin-HRP conjugate, and the presence of endogenous biotin in cercariae, cercarial bodies and adult worms was detected by transmission electron microscopy.

\section{Material and Methods}

\section{Parasites}

S. mansoni cercariae of the São Lourenço da Mata (SLM) strain were kindly supplied by Dr. J.F. Gonçalves, Sector of Malacology, Centro de Pesquisas Aggeu Magalhães, FIOCRUZ, Recife, PE. Adult worms were obtained from ether-anesthetized infected mice by heart perfusion 40-45 days after infection with approximately 150 cercariae per animal. Cercarial bodies and tails from S. mansoni were separated by vortexing (9), followed by isolation on a Percoll gradient from 10 to $50 \%$ (10).

\section{Sample solubilization}

Cercariae, cercarial bodies, cercarial tails and adult worms of S. mansoni were homogenized separately in $0.25 \mathrm{M}$ Tris- $\mathrm{HCl}$ buffer, $\mathrm{pH} 6.8$, containing $4 \%(\mathrm{w} / \mathrm{v})$ sodium dodecyl sulfate (SDS) and 10\% (v/v) 2-mercaptoethanol. After homogenization, the samples were kept at $95^{\circ} \mathrm{C}$ for $45 \mathrm{~min}$ and the digested material was used for electrophoresis and blotting. All solubilized samples were treated with $10 \%(\mathrm{w} / \mathrm{v})$ trichloroacetic acid for $15 \mathrm{~min}$ at $70^{\circ} \mathrm{C}$. The samples were then kept on ice for $30 \mathrm{~min}$ and centrifuged at $1,000 \mathrm{~g}$ for $15 \mathrm{~min}$ at room temperature. The precipitates were resuspended in $0.5 \mathrm{ml}$ phosphate buffered saline (PBS) $(0.14 \mathrm{M} \mathrm{NaCl}$, $2.7 \mathrm{mM} \mathrm{KCl}, 1.5 \mathrm{mM} \mathrm{KH}_{2} \mathrm{PO}_{4}$, and $8.2 \mathrm{mM}$ $\mathrm{Na}_{2} \mathrm{HPO}_{4}$ ), $\mathrm{pH} 7.4$, for the protein assay (11).

\section{Sample biotinylation}

A total of 2,000 cercariae, 1,500 bodies or 4,500 tails were washed with cold sterile PBS. Each sample was incubated with 0.22 $\mathrm{mM}$ biotinamidecaproate-N-hydroxysuccinimide ester (BcapNHS) (Calbiochem Boehring Diagnostics Hoechst, La Jolla, $\mathrm{CA}$ ) in $250 \mu \mathrm{l}$ PBS for $5 \mathrm{~min}$ at room temperature (12). The reaction was stopped by the addition of $500 \mu \mathrm{l} 2.2 \mathrm{mM}$ L-lysine in PBS.

\section{Detection of biotinylated polypeptides}

Solubilized samples ( $30 \mu \mathrm{g}$ protein) were submitted to SDS-PAGE (13) and the proteins were transferred to nitrocellulose sheets at a constant current of $100 \mathrm{~mA}$ for $50 \mathrm{~min}$ at room temperature. The nitrocellulose membrane was incubated overnight at $4^{\circ} \mathrm{C}$ with $3 \%(\mathrm{w} / \mathrm{v})$ skim milk in $10 \mathrm{mM}$ Tris buffer, $\mathrm{pH} 7.2$, containing $0.15 \mathrm{M} \mathrm{NaCl}$ and $0.5 \%$ (v/v) Triton X-100 (TBS-T), washed several times with TBS-T, and incubated with a streptavidin-HRP conjugate $(1: 4,000$ dilution) at room temperature. The membranes were washed with TBS-T and incubated with

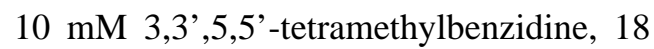
$\mathrm{mM}$ sodium dioctyl-sulfosuccinate in citrate-phosphate buffer $(50 \mathrm{mM}$ citric acid and $90 \mathrm{mM}$ disodium phosphate), $\mathrm{pH}$ 5.0, containing $25 \%(\mathrm{v} / \mathrm{v})$ ethanol, until visualization of the bands. 


\section{Electron microscopy}

For transmission electron microscopy, the cercariae, cercarial bodies and adult worms were fixed overnight in $2 \%(\mathrm{v} / \mathrm{v})$ glutaraldehyde, $4 \%(\mathrm{v} / \mathrm{v}) \mathrm{p}$-formaldehyde and $5 \mathrm{mM}$ $\mathrm{CaCl}_{2}$ in $0.1 \mathrm{M}$ cacodylate buffer, $\mathrm{pH} 7.4$, at $4^{\circ} \mathrm{C}$, postfixed with $1 \%$ (w/v) $\mathrm{OsO}_{4}$, containing $5 \mathrm{mM}$ ferricyanide, for $2 \mathrm{~h}$ at $4^{\circ} \mathrm{C}$, dehydrated in acetone and embedded in EPON 812. Sections were cut with a diamond knife, picked up on copper grids, dipped in a colloidon solution and dried. The grids were kept in PBS, pH 7.4, containing $5 \mathrm{mM}$ mannose, $5 \mathrm{mM}$ galactose and $5 \mathrm{mM}$ fucose, for $1 \mathrm{~h}$, washed with distilled water and then incubated with an avidin-colloidal gold conjugate in TBS-T for $2 \mathrm{~h}$ at $37^{\circ} \mathrm{C}$. After washing with TBS-T and distilled water, the grids were dried and incubated with uranyl acetate and lead citrate. The samples were examined with a JEOL $100 \mathrm{CX}$-II electron microscope.

\section{Results}

Cercarial bodies and tails of $S$. mansoni were separated on a 20-30\% Percoll gradient (v/v) for the tails and 40-50\% (v/v) gradient for the bodies.

Figure 1 shows the biotinylated polypeptides of cercariae, cercarial bodies and tails labeled or not with BcapNHS and identified by the streptavidin-peroxidase conjugate. Comparison of the polypeptides from labeled and unlabeled cercariae (Figure 1b and c) demonstrated the presence of several additional bands in the labeled cercariae. Polypeptides from cercarial bodies and tails isolated from labeled cercariae (Figure 1d and f) did not differ from the unlabeled samples (Figure 1e and g). However, the tails presented more biotinylated polypeptides than the bodies. When the bodies and tails were labeled after separation and isolation on the Percoll gradient, a different distribution of the biotinylated polypeptides was observed on the nitrocellulose sheets (Fig-
I

II

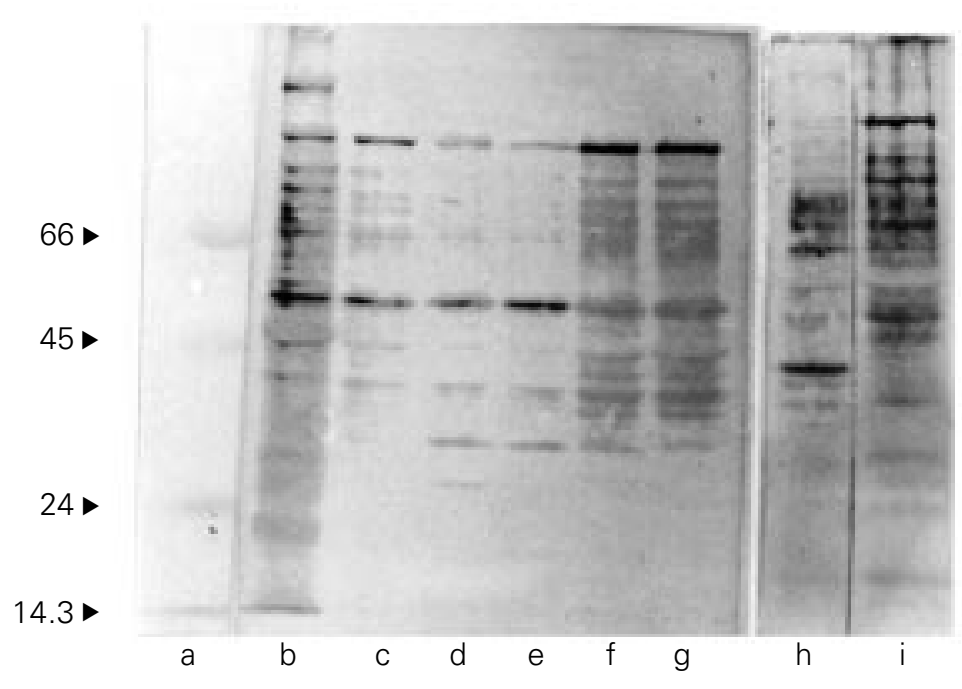

Figure 1 - Detection of biotinylated polypeptides on nitrocellulose sheets using a streptavidin-HRP conjugate. $a$, Molecular mass standards; $b$, cercariae labeled with BcapNHS; $c$, unlabeled cercariae; $d$, cercarial bodies from labeled cercariae; $e$, unlabeled bodies; $f$, cercarial tails from labeled cercariae; $g$, unlabeled tails; $h$, cercarial bodies and $i$, tails labeled with BcapNHS after isolation on a Percoll gradient.

ure $1 \mathrm{~h}$ and i) compared with the samples obtained from the labeled cercariae, including polypeptides larger than $118 \mathrm{kDa}$ and smaller than $27 \mathrm{kDa}$.

Bands were not detected on the nitrocellulose sheets in the samples containing endogenous biotinylated polypeptides incubated with HRP or streptavidin-HRP conjugate treated with BcapNHS, followed by incubation with an appropriate substrate.

Detection of the endogenous biotinylated polypeptides in male and female worms of S. mansoni by the streptavidin-HRP conjugate revealed three bands with apparent molecular masses of 59, 66 and $84 \mathrm{kDa}$ in males, and very faint bands in females (data not shown).

Biotin and biocitin were used as standards for the analysis of these compounds in cercariae. The result obtained with biotin was $13 \mu \mathrm{g}$ and the result obtained with biocitin was $14.5 \mu \mathrm{g}$ per 190,000 cercariae.

Transmission electron microscopy of all samples showed the presence of endogenous biotin in S. mansoni. In the cercariae, gold particles were mainly distributed on muscle 
Figure 2 - Transmission electron microscopy showing endogenous biotin in Schistosoma mansoni. An avidin-colloidal gold conjugate was used for the detection of the endogenous biotin. a, Muscle fibers of cercariae; $b$, spine in a cercarial body; $c$, mitochondria of cercariae; $d$, tissue of male adult worms. Magnification: 40,000X. Arrows point to gold particles.

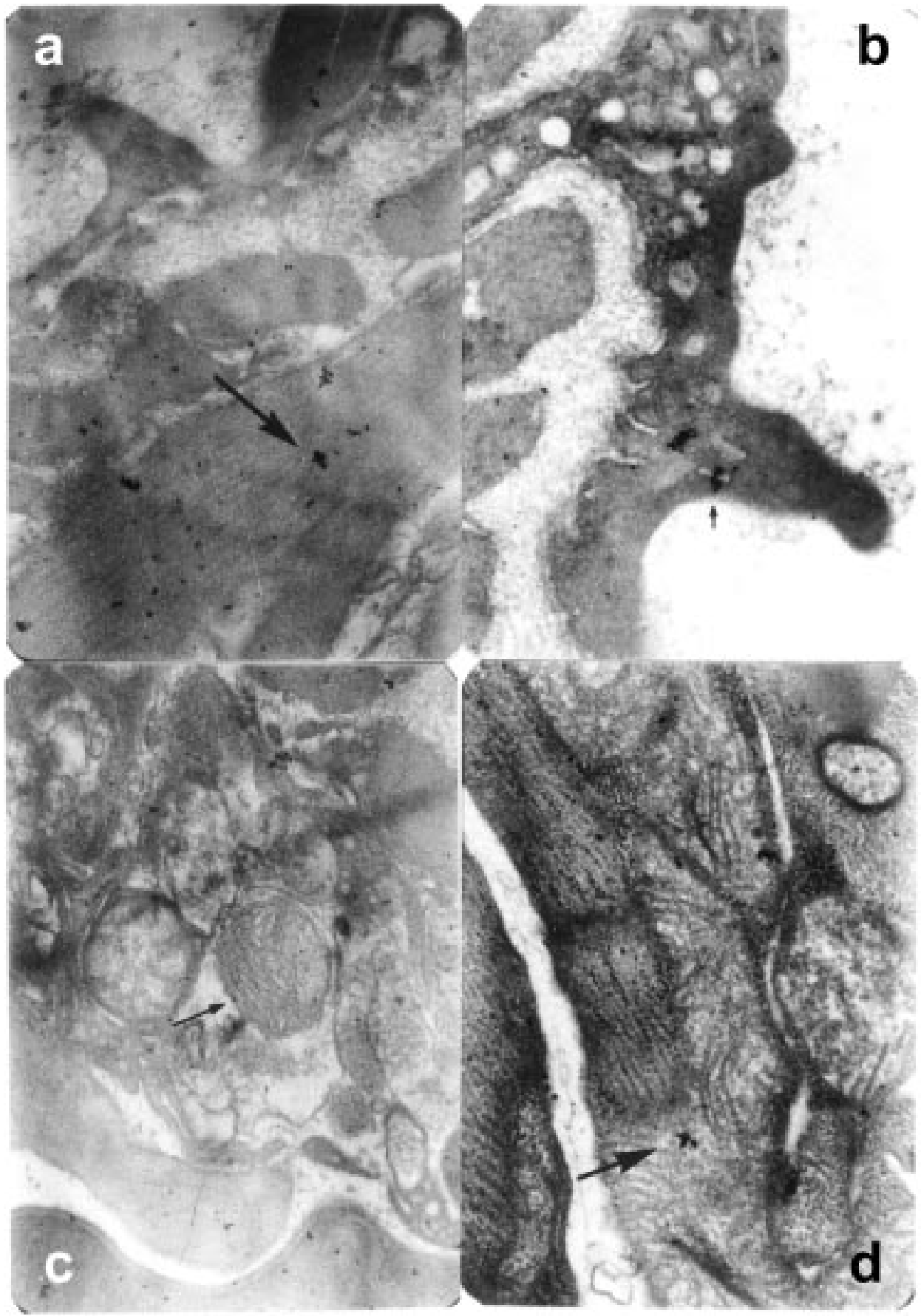

fibers (Figure 2a), but dispersed granules were observed in the tegument and mitochondria (Figure 2c). Cercarial bodies accumulated dense granules at the base of the spines (Figure 2b). In adult male worms, gold particles were concentrated in areas close to the surface of the worms (Figure 2d).

\section{Discussion}

Over the last few years, derivatives of biotin have been used to label biological macromolecules (14-16), including surface proteins of parasites. The profile of surface proteins from Brugia malayi labeled with $\mathrm{N}$ - 
hydroxysuccinimide-biotin (NHS-biotin) did not differ from that obtained with the radioiodination procedure (17). The biological function of Trypanosoma cruzi was preserved after labeling its surface proteins with sulfo-N-hydroxysuccinimide-biotin (18). In the present study, the surface of $S$. mansoni cercariae was labeled with BcapNHS in an attempt to identify antigenic proteins, without the risk involving radioactive isotopes. However, control samples presented a large number of endogenous biotinylated polypeptides.

The bands from cercarial bodies and tails obtained from labeled cercariae did not differ from those obtained from unlabeled samples, suggesting the loss of cercarial surface polypeptides from the glycocalyx during the isolation of bodies and tails, since many other bands were seen in the whole labeled cercariae. The different profile of biotinylated bands from bodies and tails labeled after their separation on a Percoll gradient may include proteins present on the external membrane of the schistosomula. The incubation with BcapNHS for 2, 4, 6 and 8 min did not alter the number of bands on the nitrocellulose sheets (data not shown), suggesting that, under the conditions of these experiments, the derivative was not internalized by the worms.
It is well known that tails consume more oxygen than bodies (19) and the transition from aerobic to anaerobic metabolism occurs only in the cercarial bodies (20). On the other hand, biotin is present in carboxylases, enzymes that catalyze the transfer of $\mathrm{CO}_{2}$ to substrates in the cells. In the host blood, the cercarial bodies find a lower oxygen tension and probably utilize anaerobic metabolism, avoiding $\mathrm{O}_{2}$ consumption and $\mathrm{CO}_{2}$ production. Under these conditions the synthesis of biotin-containing enzymes may be reduced in the parasite. The decrease in the relative amount of biotinylated polypeptides in adult worms in comparison with the cercariae may be due to an adaptive metabolic process.

Transmission electron microscopy revealed the presence of biotin in S. mansoni mainly in the muscle fibers of cercariae and cercarial bodies, but aggregates of gold particles were also observed at the base of the spines. The function of this vitamin in the parasite is unclear and needs to be investigated.

\section{Acknowledgments}

The authors wish to thank Dr. Isaíras Padovan and Rafael J.R. Padilha for help in the electron microscopy experiments.

\section{References}

1. Tielens $A G M$, van der Mer $P$, van den Heuvel JM \& van den Bergh SG (1991). The enigmatic presence of all gluconeogenic enzymes in adult Schistosoma mansoni. Parasitology, 102: 267-276.

2. Henrikson KP \& Allen SHG (1979). Purification and subunit structures of propionyl coenzyme A carboxylase Mycobacterium smegmatis. Journal of Biological Chemistry, 254: 5888-5891.

3. Henrikson KP, Allen SH \& Maloy WL (1979). An avidin monomer affinity column for the purification of biotin-containing enzymes. Analytical Biochemistry, 94: 366-370
4. Harmon FR, Berger M, Beegen $\mathrm{H} \&$ Wood HG (1980). Transcarboxylase. An electron microscopy study of the enzyme with antibodies to biotin. Journal of Biological Chemistry, 255: 9458-9464.

5. Whitney PA \& Cooper T (1973). Urea carboxylase from Saccharomyces cerevisiae. Evidence for a minimal two-step reaction sequence. Journal of Biological Chemistry, 248: 325-330.

6. Lydan MA \& O'Day DH (1991). Endogenous biotinylated proteins in Dictyostelium discoideum. Biochemical and Biophysical Research Communications, 174: 990-994.
7. Dimroth P (1982). Purification of the sodium transport enzyme oxaloacetate decarboxylase by affinity chromatography on avidin-Sepharose. FEBS Letters, 141: 5962.

8. Oei J \& Robinson BH (1985). Simultaneous preparation of the three biotin-containing mitochondrial carboxylases from rat liver. Biochimica et Biophysica Acta, 840: 1-5.

9. Ramalho-Pinto FJ, Gazzinelli G, Howells RE \& Pellegrino J (1975). Factors affecting surface changes in intact cercariae and cercarial bodies of Schistosoma mansoni. Parasitology, 71: 19-25. 
10. Lazdins JK, Stein MJ, David JR \& Sher A (1982). Schistosoma mansoni: Rapid isolation and purification of schistosomula of different developmental stages by centrifugation on discontinuous density gradients of Percoll. Experimental Parasitology, 53: 39-44.

11. Lowry OH, Rosebrough NJ, Farr AL \& Randall RJ (1951). Protein measurement with the Folin phenol reagent. Journal of Biological Chemistry, 193: 265-275.

12. Hurley $W L$, Finkelstein $E$ \& Holst BD (1985). Identification of surface proteins on bovine leucocytes by a biotin avidin protein blotting technique. Journal of Immunological Methods, 85: 195-202.

13. Laemmli UK (1970). Cleavage of structural proteins during the assembly of the head of bacteriophage T4. Nature, 227: 680-685.

14. Wilchek M \& Bayer EA (1990). Introduction to avidin-biotin technology. Methods in Enzymology, 184: 5-13.
15. Wilchek M \& Bayer EA (1990). Applications of avidin-biotin technology: literature survey. Methods in Enzymology, 184: 1445.

16. Diamandis EP \& Christopoulos TK (1991). The biotin-(strept)avidin system: Principles and applications in biotechnology. Clinical Chemistry, 37: 625-636.

17. Alvarez RM, Henry RW \& Weil GJ (1989). Use of iodogen and sulfosuccinimidobiotin to isolate cuticular proteins of filarial parasite Brugia malayi. Molecular and Biochemical Parasitology, 33: 183-190.

18. Tambourgi DV, Ogen GB, Hall BF, da-Silva WD \& Kipnis TL (1993). Biotinylation a fast and reproducible method for labelling Trypanosoma cruzi cell surface proteins. Tropical Medicine and Parasitology, 44: 91-94.
19. Von Kruger WMA, Gazzinelli G, Figueredo EA \& Pellegrino J (1978). Oxygen uptake and lactate production by Schistosoma mansoni cercariae, cercarial body and tail, and schistosomule. Comparative Biochemistry and Physiology, 60B: 41-46.

20. Horemans AMC, Tielens AGM \& van den Bergh SG (1991). The transition from an aerobic to anaerobic energy metabolism in transforming Schistosoma mansonicercariae occurs exclusively in the head. Parasitology, 109: 259-265. 